\title{
Association of Sleep Related Breathing-Disorder with Systemic Hypertension - A Cross Sectional Study from Akola, India
}

\author{
Dhondiba Haribhau Bhise ${ }^{1}$
}

${ }^{1}$ Department of Respiratory Medicine, Government Medical College, Akola, Maharashtra, India.

\section{ABSTRACT}

\section{BACKGROUND}

Hypertension or elevated blood pressure is a serious medical condition, obstructive sleep apnoea (OSA) is an important identifiable cause of hypertension. Early identification and treatment of sleep disordered breathing contributes to prevention of hypertension, and treatment of OSA may improve blood pressure control as well. This observational study was done to demonstrate the association of sleepdisordered breathing with patients who have systemic hypertension.

\section{METHODS}

Total 94 patients were included in this study. Out of ninety-four patients, eleven patients had mild sleep apnoea, nine patients had moderate sleep apnoea, and twelve had severe sleep apnoea.

\section{RESULTS}

In mild OSA there were four patients with blood pressure $<120 / 80 \mathrm{mmHg}$ while seven patients with blood pressure of $121-140 / 81$ - 90 and there were four patients with blood pressure 141 / 91. In moderate OSA there were 9, 3, 6 and 1 patients in the group of blood pressure $<120 / 80,121-140 / 81-90,>140-160 / 91-100$ and $>160 / 100 \mathrm{mmHg}$ respectively. In severe obstructive sleep apnoea there were 5, 7, 6 and 2 patients in the group of blood pressure $<120 / 80,121-140 / 81-90$, $141-160 / 91-100$ and $>160 / 100 \mathrm{mmHg}$ respectively. The severity of hypertension is strongly associated with apnoea-hypopnea index (AHI).

\section{CONCLUSIONS}

This study shows a statistically significant association of hypertension with obstructive sleep apnoea and increased severity of hypertension associated with the increased severity of obstructive sleep apnoea hypopnea syndrome (OSAHS).

\section{KEY WORDS}

Hypertension, polysomnography, apnoea / hypopnea index (AHI), Epworth sleepiness scale (ESS), Body Mass Index (BMI), obstructive sleep apnoea (OSA), obstructive sleep apnoea / hypopnea syndrome (OSAHS)
Corresponding Author: Dr. Dhondiba Haribhau Bhise, Room No 202, Second Floor Bella Vista Apartment, Near Dube STD, Geeta Nagar, Akoli br., Akola - 444001, Maharashtra, India. E-mail: bhisedhondiba@gamil.com

DOI: $10.14260 /$ jemds/2021/536

How to Cite This Article:

Bhise DH. Association of sleep related breathing - disorder with systemic hypertension - a cross sectional study from Akola, India. J Evolution Med Dent Sci 2021;10(32):2620-2623, DOI: $10.14260 /$ jemds $/ 2021 / 536$

Submission 19-02-2021, Peer Review 25-05-2021, Acceptance 02-06-2021, Published 09-08-2021.

Copyright (c) 2021 Dhondiba Haribhau Bhise. This is an open access article distributed under Creative Commons Attribution License [Attribution 4.0 International (CC BY 4.0)] 


\section{BACKGROUND}

In commensurate with most of the other guidelines it is commended that hypertension is diagnosed when a patient's systolic blood pressure (SBP) in the clinic is equal or more than $140 \mathrm{mmHg}$ and / or their diastolic blood pressure (DBP) is equal or more than $90 \mathrm{mmHg}$ following repeated examination. Hypertension is a global health problem with serious consequences. Nearly one billion people are affected by high blood pressure and still it is increasing. Untreated or uncontrolled hypertension is the single largest contributor to cardiovascular disease, causing stroke, heart failure, and coronary artery disease, and is also a major contributor to kidney disease. ${ }^{1}$ ischemic heart disease is the largest killer in the world and it contributes about $16 \%$ of the total deaths. The number of deaths from heart disease increased by more than 2 million since 2000 to nearly 8.9 million deaths in 2019.2 One of the global targets for non-communicable diseases is to reduce the prevalence of hypertension by $25 \%$ by 2025 (baseline 2010). ${ }^{3}$ Obstructive sleep apnoea (OSA) is a sleep related breathing disorder in which patient's breathing repeatedly stops and starts. It results from an obstruction of the upper airway during sleep that occurs because of inadequate motor tone of the tongue and / or airway dilator muscles, the prevalence of OSA is estimated to be around $3 \%$ to $7 \%$ in men and $2 \%$ to $5 \%$ in women. ${ }^{4}$ Obstructive sleep apnoea is a breathing disorder that has many consequences like increased risk for hypertension, cerebrovascular accidents, cardiovascular events, cognitive deterioration, and motor vehicle accidents. A canine model of sleep apnoea demonstrated that intermittent occlusion of a tracheostomy leads to the development of hypertension. Large crosssectional studies have showed that OSA is associated with hypertension.

In the Sleep Heart Health Study, the odds ratio for the presence of hypertension in the highest category of apnoea / hypopnea index (AHI) (greater than 30 events / hour) was 1.37 compared with the lowest category AHI (less than 1.5 events / hour). ${ }^{5}$ The Wisconsin Sleep Cohort Study, a prospective population-based study conducted by Peppard et al. demonstrated an increased risk of incident hypertension in patients with OSA, even at low levels of severity (AHI 5-15 events / hour). Independent of BMI, the odds ratio was 2.0 for mild (AHI 5-15 events / hour) and 2.9 for more severe sleep apnoea (AHI greater than or equal to 15 events / hour) over a 4 to 8 - year follow-up period. ${ }^{6}$ The prevalence of sleep apnoea is high in patients with drug resistant hypertension. Logan studied subjects with hypertension who were all taking optimal doses of at least three antihypertensive agents and found an OSA prevalence of 83 percent, with a mean AHI of 25 events per hour.7 The purpose of this study was to evaluate the association of sleep - disordered breathing with hypertension.

\section{METHODS}

Total 94 patients were included in this study and these patients were referred to the tertiary care hospital for evaluation of sleep disordered breathing. This cross-sectional study was conducted at Government medical college, Akola from March 2019 to February 2020.

All patients with symptoms of sleep-disordered breathing including those who were previously diagnosed to be hypertensive were included in this study.

\section{Inclusion Criteria}

1. Age equal or more than 20 years of both sexes.

2. Patients having any symptoms suggestive of Sleepdisordered breathing such as excessive daytime sleepiness, unrefreshing sleep, loud snoring, impaired concentration, frequent nocturnal awakenings, daytime fatigue, morning headache, insomnia.

3. Patients diagnosed as hypertensive when elevation in blood pressure (systolic $>140 \mathrm{mmHg}$ or diastolic $>90$ $\mathrm{mmHg}$ ) on two occasions in the absence of antihypertensive drugs.

\section{Exclusion Criteria}

1. Screening blood pressure $>180 / 110$ which would require definitive therapy.

2. Recent co-morbid conditions like cerebrovascular disease, stroke, coronary artery disease, congestive heart failure, atrial fibrillation, renal disease.

Thorough clinical evaluation including history from bed partner and physical examination were done. Patients were asked the questions related to sleep apnoea, like loud snoring, difficulty in paying attention while awake, Sleepiness or lack of energy during the day, sleepiness while driving, excessive daytime sleepiness, irritability, and the Epworth sleepiness scale (ESS) was used to interpret the daytime sleepiness. ENT specialist consultation for evaluation was done in patients who had symptoms of upper respiratory tract abnormality. ${ }^{8} \mathrm{~A}$ meticulous respiratory system examination was carried out. when patient was relaxed. Blood pressure was measured from right arm in sitting position; when patient had rested calmly for at least 15 minutes and the blood pressure was monitored on two different times by using sphygmomanometer approximately 5 to 7 days apart. The head and neck physical examination was carried out for the diagnosis of obstructive sleep apnoea; using standard equipment, weight and height of all those patients who were involved in the study were measured. The Body mass index (BMI) is a patient's body mass divided by the square of the body height in meters. A standard instrument (Epworth Sleepiness Scale) is used to measure daytime sleepiness and a value above 10 is considered abnormal, although the lower score does not exclude it.

\section{Polysomnography $9,10,11$}

An overnight polysomnography is performed in sleep laboratory using Compumedics E-Series PSG System. Parameters used to monitor:

- $\quad$ Electrooculogram (EOG): Electrodes are attached around the eyes because electrodes pick up the inherent voltage within the eye and it is also used to record eye movements during polysomnography.

- Pulse Oximeter: During overnight polysomnography pulse oximeter measures oxygen saturation level or the 
oxygen levels in patients' blood. Equal or more than $30 \%$ of the recording time with oxygen saturation less than 90 $\%$ by pulse oximeter is called as nocturnal desaturation.

- Electrocardiogram (ECG): An electrocardiogram measures your heart's electrical activity.

- Electromyography (EMG): It is used to record skeletal muscles activity (chin muscle).

- Thoracic and abdominal bands record thoracic and abdominal movements during polysomnography.

- $\quad$ Airflow sensors: They measure respiratory rate and flow in general purpose thermistor as the temperature sensors.

- Body position sensor: They are used for detecting the sleep position during polysomnography.

- Periodic leg movement (PLM) sensors: It is used for monitoring of periodic leg movements (PLM). It also helps to diagnose restless leg syndrome (RLS).

\section{Statistical Analysis}

All the data required for this study were collected and analysed statistically to determine the association between these variables using the Statistical Package for the Social Sciences version 20.0 Software, the means and proportions were compared by analysing the Variance and chi square test, which has shown the association between apnoea - hypopnea index (AHI) and systemic hypertension. Chi-square test was significant ( $P$ - 0.0078) which shows that both these factors hypertension and $\mathrm{AHI}$ are statistically significant.

\section{RESULTS}

This study shows the association of patients who have sleep disordered breathing with hypertension. The severity of obstructive sleep apnoea is measured by the number of apnoea plus hypopnea events in one hour of sleep An apnoea - hypopnea index (AHI) greater than 5 events in one hour is suggestive of OSA, if it is between five and 14 times per hour is defined as mild sleep apnoea. If it is between 15 and 29 events is rated as moderate sleep apnoea, and the presence of 30 or more than 30 events per night is classified as severe sleep apnoea.

\begin{tabular}{|c|c|c|c|c|}
\hline BP AHI & $<5$ & $5-14$ & $15-29$ & 30 \\
\hline Yes & $21(22.3 \%)$ & $11(11.7 \%)$ & $9(9.5 \%)$ & $12(12.7 \%)$ \\
\hline No & $29(30.8 \%)$ & $6(6.3 \%)$ & $5(5.3 \%)$ & $1(1.0 \%)$ \\
\hline \multicolumn{5}{|c|}{ Table. 1. Association of AHI with Blood Pressure } \\
\hline
\end{tabular}

The presence of pre-existing pre-diagnosed medical condition like hypertension and its severity was taken into consideration while reviewing the severity of obstructive sleep apnoea. There was a significant association of blood pressure with apnoea / hypopnea index. Patients with higher blood pressure had higher AHI values. High blood pressure being defined as more than 140 / $90 \mathrm{~mm}$ of $\mathrm{Hg}$. Eleven patients (i.e.11.7 \%) had AHI values between 5 - 14, 9 patients (9.5\%) had values between 15 - 29 and 12 patients (12.7\%) had AHI more than 30 . Only one patient in non-hypertensive group had AHI more than 30.

While 7 patients had systolic blood pressure between 121 and 140 and diastolic within 81 - 90, whereas there were 4 patients with blood pressure 141 / 91. In moderate obstructive sleep apnoea group (AHI 15 - 29) there were 9, 3, 6 and 1 patients in the group of blood pressure $<120 / 80,121$ to 140 systolic and diastolic 81 to $90,141-160 / 91$ - 100 and $>160 / 100$ respectively. In severe group (AHI > 30) there were $5,7,6$ and 2 patients in the group of blood pressure $<120$ / 80, 121 to 140 systolic and diastolic 81 to 90,141 - 160 / 91 - 100 and $>160 / 100$ respectively. The chi-square test was significant $(P-0.02)$ which indicated the severity of hypertension strongly associated with AHI.

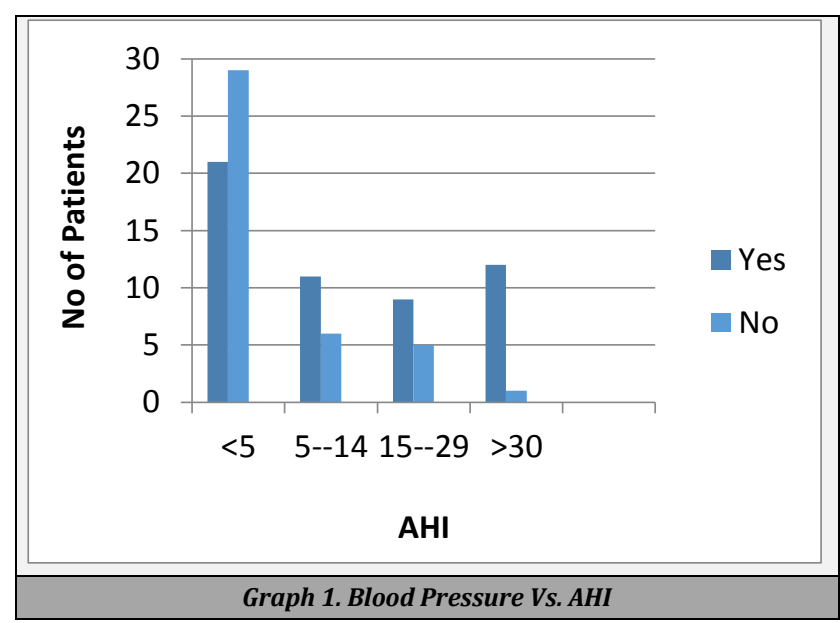

\begin{tabular}{|ccccc|}
\hline HTN AHI & $<\mathbf{5}$ & $\mathbf{5 - 1 4}$ & $\mathbf{1 5 - 2 9}$ & $>\mathbf{3 0}$ \\
$\quad \begin{array}{c}\text { Sys }<120 \\
\text { Dia }<80\end{array}$ & $27(28.7 \%)$ & $4(4.2 \%)$ & $9(9.5 \%)$ & $5(5.3 \%)$ \\
$\begin{array}{c}\text { Sys } 121-140 \\
\text { Dia } 81-90\end{array}$ & $7(7.4 \%)$ & $7(7.4 \%)$ & $3(3.1 \%)$ & $7(7.4 \%)$ \\
$\begin{array}{c}\text { Sys } 141-160 \\
\text { Dia } 91-100\end{array}$ & $6(6.3 \%)$ & $4(4.2 \%)$ & $6(6.3 \%)$ & $6(6.3 \%)$ \\
$\quad \begin{array}{l}\text { Sys }>160 \\
\text { Dia }>100\end{array}$ & 0 & 0 & $1(1 \%)$ & $2(2.1 \%)$ \\
Table 2. Association of AHI with the Severity of Blood Pressure. \\
\hline
\end{tabular}

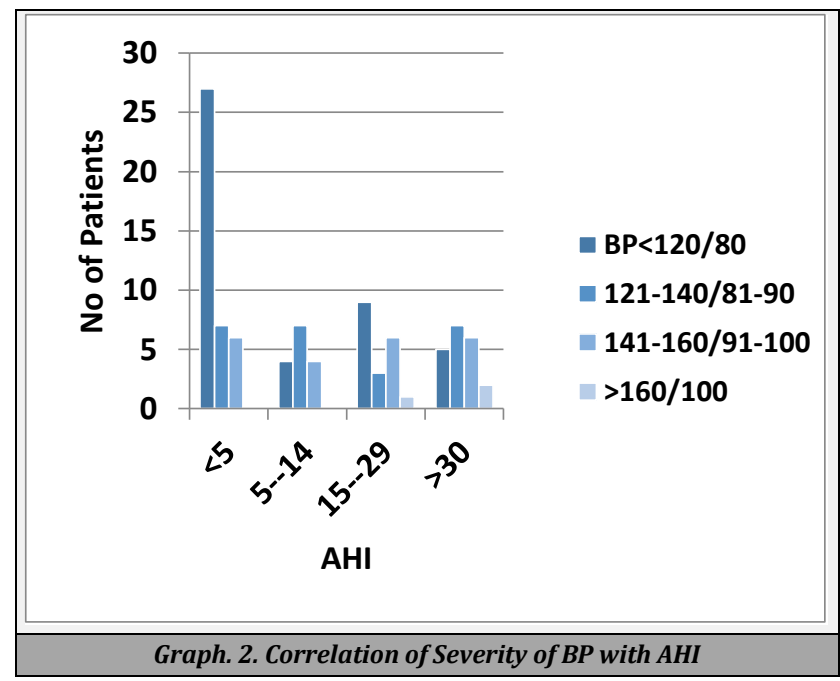

\section{DISCUSSION}

Hypertension was assumed if antihypertensive drugs were being prescribed or if the blood pressure was detected above 140 / $90 \mathrm{mmHg}$ at different occasions. This study provided data on association of sleep-disordered breathing and its consequence like hypertension. Obstructive sleep apnoea (OSA) is diagnosed by using apnoea-hypopnea index (AHI) 
which is defined as the sum of the number of apnoeas and hypopneas per hour of sleep.

When patient had symptoms like excessive daytime somnolence, chronic fatigue, and unrefreshing sleep with AHI greater than 5 to 10 events per hour then obstructive sleep apnoea - hypopnea syndrome (OSAHS) was said to be present in our study. In this study we have taken 5, 14, 29 and above cut off points of AHI to indicate mild, moderate, and severe levels of OSAHS respectively. Several other investigators have taken different levels of AHI cut off points to indicate three different levels of OSAHS. Obstructive sleep apnoea is an important identifiable cause of hypertension. There is linear association between obstructive sleep apnoea and hypertension. Increase in the repetitive episodes of airflow reduction (hypopnea) or cessation (apnoea) increases the probability of hypertension. In this study $34 \%$ of hypertensive patients showed AHI more than 5 than non-hypertensive patients. In this study it was shown that relationship between OSA and hypertension was affected by factors like age, overweight, and apnoea.

Christopher John, Mathew Thomas have shown that there is a relationship between OSA and HT that, although partially explained by the confounding variables in their study. $\mathrm{J} T$ Carlson et al. concluded that BMI, age and SA were recognized as independent risk factors of hypertension. José M Marin et al. concluded that patients with OSA were related with increased adjusted risk of incident hypertension than that of patients without OSA. An increased prevalence of sleep disordered breathing and hypertension was compared with patients not having hypertension. Our study shows the association between increase in the body weight and the risk of OSA. Decrease in the BMI diminished the relationship between obstructive sleep apnoea and hypertension. The relationship between sleep disordered breathing and hypertension depends on two or more variables. There is simple linear regression between sleep disordered breathing and hypertension. In the given observational study, probability due to independent variables or unrevealed addle cannot be excluded. The association between SDB and hypertension in a large cohort of middle-aged and older persons was found in the study of $\mathrm{F}$ J Nieto et al. from the largest cross-sectional study. Babak Mokhlesi et al. findings showed significant correlation between REM OSA and development of hypertension. Khin Mae Hla, Terry B. Young et al. found the association between sleep apnoea and hypertension independent of age, sex, and obesity in a nonelected, community-based adult population. A number of studies have achieved a reduction in blood pressure by relieving OSA with nasal continuous positive airway pressure suggesting that there is a causal link. The successful treatment of sleep apnoea and hypertension depends on causative factors, levels of blood pressure and severity of sleep apnoea. Patients with OSA and hypertension are hypothesized to occur primarily due to sympathetic nervous system over activity. Negative intrathoracic pressure and overnight intermittent hypoxia lead to chemoreceptor activation and increased sympathetic outflow. Nocturnal intermittent hypoxia during sleep in OSA results in increased oxidative stress, systemic inflammation and endothelial dysfunction that leads to vascular remodelling, and atherosclerosis. These changes predispose the patients to hypertension, and the hypertension leads to endothelial dysfunction and vascular remodelling. So we concise that our study shows strong association between systemic hypertension and sleep disordered breathing.

\section{CONCLUSIONS}

Our study concluded that obstructive sleep apnoea significantly impacts blood pressure with a strong statistically significant association of hypertension with obstructive sleep apnoea. Increase severity of hypertension associates with the severity of obstructive sleep apnoea-hypopnea syndrome. Early management of obstructive sleep apnoea prevents the development of hypertension.

Data sharing statement provided by the authors is available with the full text of this article at jemds.com.

Financial or other competing interests: None.

Disclosure forms provided by the authors are available with the full text of this article at jemds.com.

\section{REFERENCES}

[1] Fisher NDL, Curfman G. Hypertension-a public health challenge of global proportions. JAMA 2018;320(17):1757-9.

[2] https://www.who.int/news-room/fact-sheets/detail or who global health estimates.

[3] WHO. Global action plan for the prevention and control of NCDs 2013-2020. Global Strategy 2013.

[4] Punjabi NM. The epidemiology of adult obstructive sleep apnoea. Proc Am Thorac Soc 2008;5(2):136-43.

[5] Nieto FJ, Young TB, Lind BK, et al. Association of sleepdisordered breathing, sleep apnoea and hypertension in a large community-based study. Sleep Heart Health Study. JAMA 2000;283(14):1829-36.

[6] Peppard PE, Young T, Palta M, et al. Prospective study of the association between sleep-disordered breathing and hypertension. N Engl J Med 2000;342(19):1378-84.

[7] Logan AG, Perlikowski SM, Mente A, et al. High prevalence of unrecognized sleep apnoea in drug-resistant hypertension. J Hypertens 2001;19(12):2271-7.

[8] Johns MW. A new method for measuring daytime sleepiness: the epworth sleepiness scale. Sleep 1991;14(6):540-5.

[9] Lerman SE, Eskin E, Flower DJ, et al. Fatigue risk management fatigue risk management in the workplace. J Occup Environ Med 2012;54(2):231-58.

[10] Kales A, Rechtschanffen AA. A manual standardized terminology, techniques and scoring system for sleep stages of human subjects. Washington, DC: United States Government Printing service 1968.

[11] Berrry RB, Brooks R, Gamaldo CE, et al. The AASM manual for the scoring of sleep and associated events: rules terminology and technical specifications. Version 2.0. Darien, IL; American Academy of Sleep Medicine 2012. 